\author{
MITSUBISHI ELECTRIC RESEARCH LABORATORIES \\ http://www.merl.com
}

\title{
Global Optimization of Multi-period Optimal Power Flow
}

\author{
Gopalakrishnan, A.; Raghunathan, A.U.; Nikovski, D.; Biegler, L.T.
}

TR2013-066 June 2013

\begin{abstract}
In this work, we extend the algorithm proposed in [1] to solve multi-period optimal power flow (MOPF) problems to global optimality. The multi-period version of the OPF is time coupled due to the integration of storage systems into the network, and ramp constraints on the generators. The global optimization algorithm is based on the spatial branch and bound framework with lower bounds on the optimal objective function value calculated by solving a semidefinite programming (SDP) relaxation of the MOPF. The proposed approach does not assume convexity and is more general than the ones presented previously for the solution of MOPF. We present a case study of the IEEE 57 bus instance with a time varying demand profile. The integration of storage in the network helps to satisfy loads during high demands and the ramp constraints ensure smooth generation profiles. The SDP relaxation does not satisfy the rank condition, and our optimization algorithm is able to guarantee global optimality within reasonable computational time.
\end{abstract}

American Control Conference (ACC)

This work may not be copied or reproduced in whole or in part for any commercial purpose. Permission to copy in whole or in part without payment of fee is granted for nonprofit educational and research purposes provided that all such whole or partial copies include the following: a notice that such copying is by permission of Mitsubishi Electric Research Laboratories, Inc.; an acknowledgment of the authors and individual contributions to the work; and all applicable portions of the copyright notice. Copying, reproduction, or republishing for any other purpose shall require a license with payment of fee to Mitsubishi Electric Research Laboratories, Inc. All rights reserved. 



\title{
Global Optimization of Multi-period Optimal Power Flow
}

\author{
Ajit Gopalakrishnan ${ }^{1,2}$, Arvind U. Raghunathan ${ }^{2}$, Daniel Nikovski ${ }^{2}$ and Lorenz T. Biegler ${ }^{1}$
}

\begin{abstract}
In this work, we extend the algorithm proposed in [1] to solve multi-period optimal power flow (MOPF) problems to global optimality. The multi-period version of the OPF is time coupled due to the integration of storage systems into the network, and ramp constraints on the generators. The global optimization algorithm is based on the spatial branch and bound framework with lower bounds on the optimal objective function value calculated by solving a semidefinite programming (SDP) relaxation of the MOPF. The proposed approach does not assume convexity and is more general than the ones presented previously for the solution of MOPF. We present a case study of the IEEE 57 bus instance with a time varying demand profile. The integration of storage in the network helps to satisfy loads during high demands and the ramp constraints ensure smooth generation profiles. The SDP relaxation does not satisfy the rank condition, and our optimization algorithm is able to guarantee global optimality within reasonable computational time.
\end{abstract}

\section{INTRODUCTION}

Electric power grids worldwide are currently undergoing radical changes in the face of increasing demand and uncertainty caused by the integration of intermittent renewable energy sources, and deregulation of the industry [2], [3], [4]. Distributed energy storage is considered to be an effective approach for addressing the operational challenges associated with these trends [5]. Grid-integrated storage technologies can defer the need for new electricity capacity, improve load following, provide spinning reserve, correct frequency, voltage, and power factors [6]. Although the benefits of such storage schemes are widely accepted, the appropriate storage technology along with the required capacity and rates of charge/discharge are a continuing research topic, see e.g. [7]. Some important criteria for an effective storage strategy are: (i) dispatchability response to fluctuations in electricity demand; (ii) interruptibility reaction to the intermittency in energy supplies like wind and solar; and (iii) efficiency in recovering energy that is otherwise wasted [8].

The Optimal Power Flow (OPF) problem for alternating current (AC) circuits concerns the problem of determining bus voltages and generator power levels to minimize a cost function. The cost functions employed include generator cost, resistive losses or tertiary voltage control. The constraints for OPF include (i) the AC power flow constraints, (ii) bounds on power generation, (iii) bounds on bus voltage magnitudes, (iv) bounds on thermal losses, and (v) limits on power transfer on lines. The OPF problem, first introduced by Carpentier [9], is a nonconvex optimization problem

\footnotetext{
${ }^{1}$ Department of Chemical Engineering, Carnegie Mellon University, Pittsburgh, PA 15213. \{agopalak, lb01\} @andrew. cmu.edu

2 Mitsubishi Electric Research Laboratories, Cambridge,MA 02139. \{raghunathan, nikovski\}@merl.com
}

with quadratic constraints and quadratic objective function (QCQP). In recent years, considerable progress has been made in obtaining globally optimal solutions to the OPF. We provide a short survey of the recent progress on the OPF with emphasis on papers related to MOPF.

\section{A. Literature Survery}

A survey of the approaches for solving the OPF prior to 2008 are provided in [10], [11], [12]. Recently there has been an effort at obtaining globally optimal solutions to the nonconvex problems through second order cone programming (SOCP) and SDP relaxations. We refer the interested reader to works of Jabr [13], [14], [15], Sojoudi and Lavaei [16], Farivar and Low [17] and Lavaei et. al. [18] on using SOCP. SDP relaxation were employed by Bai and co-workers in [19], [20]; Low and co-workers in [21], [22] and Tse and co-workers in [23].

All of the above approaches can show zero duality gap under the satisfaction of sufficient conditions for their relaxations. The sufficient conditions (rank condition) only hold under restrictive assumptions on the network topology and constraints on the OPF. Further, the above approaches provide no recourse when sufficient conditions are not satisfied. The work of Lesieutre et al [24] and Gopalakrishnan et al [1] provide examples that fail to satisfy the sufficient conditions.

Recently the authors [1] proposed to solve the OPF problem using a branch and bound (B \& B) algorithm with SDP based lower bounds. The proposed approach does not make assumptions on the network topology or the type of bounds. It was also shown that this approach outperforms the Lagrangian duality based B \& B approach suggested by Phan [25].

Gayme and Topcu [26] considered OPF problems with storage by extending the work of Lavaei and Low [21] to multiple periods. Warrington et al [27] consider the solution of MOPF problems using a Lagrangian dual based decomposition, in the presence of storage and ramp constraints. Both of these papers assume the satisfaction of the sufficient conditions for SDP formulations of the OPF problem.

\section{B. Our Contribution}

In this work, we are interested in extending the algorithm in [1] for the solution of MOPF. The MOPF is time coupled due to the presence of storage at various buses in the network. Further, we also consider ramp constraints on the power generation at buses. As a result, we obtain a series of OPF problems for different times that are coupled through the storage difference equation and ramps between successive time points. We employ a B \& B algorithm with SDP 
relaxation providing lower bounds to solve the MOPF. This has been shown to work well in the context of OPF problems [1]. In contrast to recent approaches of [26], [27], we do not make any restrictive assumptions on the satisfaction of the rank condition. In spite of the generality, the algorithm is computationally efficient and the approach holds promise.

The paper is organized as follows. Section II formulates the MOPF problem and also its SDP relaxation. Section III outlines our SDP based B \& B algorithm. Section IV provides the results and some directions for future work.

\section{Notation}

In the following, we use $\mathrm{j}$ to denote the imaginary root of -1 . For a complex variable $z, \operatorname{Re}(z)$ and $\operatorname{Im}(z)$ will denote the real and imaginary parts of the complex variable $z ;|z|$ will denote the magnitude of the complex variable and $z^{*}$ will denote the Hermitian conjugate. For a symmetric matrix $A$ its trace will be denoted as $\operatorname{Tr}(A)$ which is the sum of the diagonal entries.

\section{MOPF PROBLEM}

We start with a given graph $\mathcal{G}=(\mathcal{N}, \mathcal{E})$ of the power network, where the nodes $i \in \mathcal{N}, j \in \mathcal{N}$ represent the buses and the edges in $\mathcal{E}$ represent branches connecting an ordered pair of buses $(i, j)$. $\mathcal{N}^{G} \subseteq \mathcal{N}$ denotes the set of buses connected to generators. Further we introduce $\mathcal{L}$ to denote the set in which the edges in $\mathcal{E}$ are duplicated, i.e. $(i, j) \in \mathcal{E} \Rightarrow(i, j),(j, i) \in \mathcal{L}$. For a bus $i, k \sim i$ denotes the set of buses $k$ which are connected to $i$. The line admittance is given by $y_{i j}=g_{i j}+\mathrm{j} b_{i j},(i, j) \in \mathcal{L}$, with $y_{i j}=0$ for $i \not j$. The bus admittance matrix, which relates the current injections to the bus voltages, is formed as

$$
Y_{\text {bus }, i}= \begin{cases}-y_{i j}, & i \neq j \\ y_{i i}+\sum_{k \sim i} y_{i k}, & i=j\end{cases}
$$

where $y_{i i}$ denotes the admittance-to-ground at bus $i$.

We are interested in solving the multi-period OPF problem over time periods $t \in \mathcal{T}=\{1, \cdots, T\}$. Let $\mathbf{v}(t)=\left(V_{1}(t), V_{2}(t), \cdots, V_{|\mathcal{N}|}(t)\right)$ denote the complex bus voltages, and $\mathbf{i}=\left(I_{1}(t), I_{2}(t), \cdots, I_{|\mathcal{N}|}(t)\right)$ denote the bus injection currents. Then by Ohm's and Kirchoff's laws applied on the entire network, $\mathbf{i}(t)=$ $Y_{\text {bus }} \mathbf{v}(t)$. Let $\mathbf{P}^{G}(t)=\left(P_{1}^{G}(t), \cdots, P_{|\mathcal{N}|}^{G}(t)\right), \mathbf{Q}^{G}(t)=$ $\left(Q_{1}^{G}(t), \cdots, Q_{|\mathcal{N}|}^{G}(t)\right)$ denote the power generations at each bus, with $\left(P_{i}^{G}(t), Q_{i}^{G}(t)\right)=0, \forall i \notin \mathcal{N}^{G}, \forall t \in \mathcal{T}$. The power flowing from bus $i$ to $j$ is calculated as, $S_{i j}(t)=$ $P_{i j}(t)+\mathrm{j} Q_{i j}(t)=V_{i}(t)\left|V_{i}(t)-V_{j}(t)\right|^{*} y_{i j}^{*}$.

Further, let $\mathcal{N}^{S} \subseteq \mathcal{N}$ denote the set of buses with storage capability. Let $\mathbf{B}(t)=\left(B_{1}(t), \cdots, B_{|\mathcal{N}|}(t)\right)$ denote the energy stored and $\mathbf{R}(t)=\left(R_{1}(t), \cdots, R_{|\mathcal{N}|}(t)\right)$, the rate of charge/discharge of real power to/from storage at each bus, with $\left(B_{i}(t), R_{i}(t)\right)=0, \forall i \notin \mathcal{N}^{S}, \forall t \in \mathcal{T}$.
The multiperiod OPF problem with storage (MOPF) can be stated as,

$$
\begin{aligned}
& \min \sum_{t=1}^{t=T} \sum_{i \in \mathcal{N}^{G}} c_{2 i}\left(P_{i}^{G}(t)\right)^{2}+c_{1 i} P_{i}^{G}(t)+c_{0 i} \\
& \text { s.t. } P_{i}^{G}(t)-P_{i}^{D}(t)-R_{i}(t)=\mathbf{x}(t)^{T} Y_{i} \mathbf{x}(t), i \in \mathcal{N} \\
& Q_{i}^{G}(t)-Q_{i}^{D}(t)=\mathbf{x}(t)^{T} \bar{Y}_{i} \mathbf{x}(t), i \in \mathcal{N} \\
& P_{i j}(t)=\mathbf{x}(t)^{T} Y_{i j} \mathbf{x}(t),(i, j) \in \mathcal{L} \\
& Q_{i j}(t)=\mathbf{x}(t)^{T} \bar{Y}_{i j} \mathbf{x}(t),(i, j) \in \mathcal{L} \\
& P_{i}^{\min } \leq P_{i}^{G}(t) \leq P_{i}^{\max }(t), i \in \mathcal{N}^{G} \\
& Q_{i}^{\min } \leq Q_{i}^{G}(t) \leq Q_{i}^{\max }, i \in \mathcal{N}^{G} \\
& \left(V_{i}^{\min }\right)^{2} \leq \mathbf{x}(t)^{T} M_{i} \mathbf{x}(t) \leq\left(V_{i}^{\max }\right)^{2}, i \in \mathcal{N} \\
& P_{i j}^{2}(t)+Q_{i j}^{2}(t) \leq\left(S_{i j}^{\max }\right)^{2},(i, j) \in \mathcal{L} \\
& \mathbf{x}(t)^{T} M_{i j} \mathbf{x}(t) \leq L_{i j}^{\max },(i, j) \in \mathcal{L} \\
& B_{i}^{\min } \leq B_{i}(t) \leq B_{i}^{\max }, i \in \mathcal{N}^{S} \\
& R_{i}^{\min } \leq R_{i}(t) \leq R_{i}^{\max }, i \in \mathcal{N}^{S} \\
& B_{i}(t)=B_{i}(t-1)+\eta R_{i}(t), i \in \mathcal{N}^{S} \\
& B_{i}(0)=B_{i}^{0}, B_{i}(T) \geq B_{i}^{*}, i \in \mathcal{N}^{S} \\
& \left.\begin{array}{l}
\Delta P_{i}^{\min } \leq P_{i}^{G}(t+1)-P_{i}^{G}(t), \\
P_{i}^{G}(t+1)-P_{i}^{G}(t) \leq \Delta P_{i}^{\max }
\end{array}\right\} i \in \mathcal{N}^{G} \\
& \left.\begin{array}{l}
\Delta Q_{i}^{\min } \leq Q_{i}^{G}(t+1)-Q_{i}^{G}(t), \\
Q_{i}^{G}(t+1)-Q_{i}^{G}(t) \leq \Delta Q_{i}^{\max }
\end{array}\right\} i \in \mathcal{N}^{G}
\end{aligned}
$$

where $\mathbf{x}(t):=\left[\operatorname{Re}(\mathbf{v}(t))^{T} \operatorname{Im}(\mathbf{v}(t))^{T}\right]^{T}$. Equations (2a) (21) apply for $\forall t \in \mathcal{T}$ and Equations (2n)-(2o) for $\forall t \in$ $\mathcal{T} /\{T\}$. The matrices $Y_{i}, \bar{Y}_{i}, Y_{i j}, \bar{Y}_{i j}, M_{i}$ are as defined in Appendix I. Equations (2a), (2b) represent the real and reactive power balances at each bus. Equations $(2 \mathrm{c}),(2 \mathrm{~d})$ are the real and reactive branch power flows, which are limited by constraint $(2 \mathrm{~h})$. Constraints $(2 \mathrm{e}),(2 \mathrm{f})$ and $(2 \mathrm{n}),(2 \mathrm{o})$ are limits on power generation and ramps on power generation respectively. $(2 \mathrm{~g})$ is the voltage magnitude limit and $(2 \mathrm{i})$ is the thermal limit. Constraints $(2 \mathrm{j})$ represents a bound on the energy stored, and (2k) is a bound on rate of charge/discharge of energy from the storage system. In (2l) the parameter $\eta$ represents the round trip efficiency of the storage system. Constraint $(2 \mathrm{~m})$ represents the initial and terminal condition for the above multi-period optimization problem. Since we are approximating an infinite horizon problem by a finite horizon solution, the terminal constraint is necessary to avoid the problem of storage depletion. This helps to ensure that there is sufficient energy to meet future requirements beyond the time horizon considered, and avoids a myopic solution. Only the constraints (2l), (2n), (2o) are coupled in time, while the rest of the model is decoupled in time.

\section{A. SDP Relaxation of $M O P F$}

We introduce the following convex formulation with a lifting of the quadratic voltage terms $\mathbf{x}(t) \mathbf{x}^{T}(t)$ to a $2|\mathcal{N}| \times$ $2|\mathcal{N}|$ symmetric matrix $\mathbf{W}(t)$ while retaining the remaining variables. This is done so as to only relax the variables that appear in a nonconvex fashion in the constraints. Further, we eliminate the line flow variables $P_{i j}(t), Q_{i j}(t)$ to obtain 
a more compact formulation. However, the apparent power line limit constraint (2h) will have to reformulated to a second order cone constraint as shown in (3k). Dropping the $\operatorname{rank}(\mathbf{W}(t))=1$ condition, we formulate the SDP relaxation as follows,

$$
\begin{aligned}
& \min \sum_{t=1}^{t=T} \sum_{i \in \mathcal{N}^{G}} a_{i}(t) \\
& \text { s.t. } \mathbf{W}(t) \succeq 0 \\
& \forall i \in \mathcal{N}^{G} \text { : } \\
& {\left[\begin{array}{cc}
a_{i 0}(t) & a_{i 1}(t) \\
a_{i 1}(t) & -1
\end{array}\right] \preceq 0, \quad\left[\begin{array}{cc}
1 & r_{i}^{1}(t) \\
r_{i}^{1}(t) & r_{i}^{2}(t)
\end{array}\right]} \\
& P_{i}^{\min } \leq P_{i}^{G}(t) \leq P_{i}^{\max }, \quad\left[\underline{\lambda}_{i}^{P}(t), \bar{\lambda}_{i}^{P}(t)\right] \\
& Q_{i}^{\min } \leq Q_{i}^{G}(t) \leq Q_{i}^{\max }, \quad\left[\underline{\lambda}_{i}^{Q}(t), \bar{\lambda}_{i}^{Q}(t)\right] \\
& \left.\begin{array}{l}
\Delta P_{i}^{\min } \leq P_{i}^{G}(t+1)-P_{i}^{G}(t), \\
P_{i}^{G}(t+1)-P_{i}^{G}(t) \leq \Delta P_{i}^{\max }
\end{array}\right\} \quad\left[\begin{array}{c}
\rho_{i}(t) \\
\bar{\rho}_{i}(t)
\end{array}\right] \\
& \left.\begin{array}{l}
\Delta Q_{i}^{\min } \leq Q_{i}^{G}(t+1)-Q_{i}^{G}(t), \\
Q_{i}^{G}(t+1)-Q_{i}^{G}(t) \leq \Delta Q_{i}^{\max }
\end{array}\right\} \quad\left[\begin{array}{l}
\underline{\sigma}_{i}(t) \\
\bar{\sigma}_{i}(t)
\end{array}\right] \\
& \forall i \in \mathcal{N}: \\
& P_{i}^{G}(t)-P_{i}^{D}(t)-R_{i}(t)=\operatorname{Tr}\left(Y_{i} \mathbf{W}(t)\right), \quad\left[\alpha_{i}(t)\right] \\
& Q_{i}^{G}(t)-Q_{i}^{D}(t)=\operatorname{Tr}\left(\bar{Y}_{i} \mathbf{W}(t)\right), \\
& {\left[\beta_{i}(t)\right]} \\
& \left.\begin{array}{l}
\left(V_{i}^{\min }\right)^{2} \leq \operatorname{Tr}\left(M_{i} \mathbf{W}(t)\right), \\
\operatorname{Tr}\left(M_{i} \mathbf{W}(t)\right) \leq\left(V_{i}^{\max }\right)^{2}
\end{array}\right\} \\
& {\left[\begin{array}{l}
\lambda_{i}^{V}(t) \\
\bar{\lambda}_{i}^{V}(t)
\end{array}\right]} \\
& \forall(i, j) \in \mathcal{L}: \\
& \left\|\begin{array}{l}
\operatorname{Tr}\left(Y_{i j} \mathbf{W}(t)\right) \\
\operatorname{Tr}\left(\bar{Y}_{i j} \mathbf{W}(t)\right)
\end{array}\right\|_{2} \leq S_{i j}^{\max }, \quad\left[\left\|\begin{array}{c}
r_{i j}^{1}(t) \\
r_{i j}^{2}(t)
\end{array}\right\|_{2} \leq \lambda_{i j}(t)\right] \\
& \operatorname{Tr}\left(M_{i j} \mathbf{W}(t)\right) \leq L_{i j}^{\max }, \\
& {\left[\mu_{i j}(t)\right]} \\
& \forall i \in \mathcal{N}^{S} \text { : } \\
& B_{i}^{\min } \leq B_{i}(t) \leq B_{i}^{\max }, \\
& {\left[\underline{\lambda}_{i}^{B}(t), \bar{\lambda}_{i}^{B}(t)\right](3 \mathrm{~m})} \\
& R_{i}^{\min } \leq R_{i}(t) \leq R_{i}^{\max }, \\
& B_{i}(0)=B_{i}^{0}, B_{i}(T) \geq B_{i}^{*}, \\
& {\left[\underline{\lambda}_{i}^{R}(t), \bar{\lambda}_{i}^{R}(t)\right] \quad(3 \mathrm{n})} \\
& B_{i}(t)=B_{i}(t-1)+\eta R_{i}(t)
\end{aligned}
$$

where,

$$
a_{i 0}(t)=c_{i 0}+c_{i 1} P_{i}^{G}(t)-a_{i}(t), a_{i 1}(t)=\sqrt{c_{i 2}} P_{i}^{G}(t)
$$

The dual multipliers corresponding to the constraints in the SDP relaxation are indicated in brackets [.]. Note that for constraint in (3c) the multiplier is a matrix while for $(3 \mathrm{k})$ the mulitplier is expressed as the dual second order cone. Though the SDP solvers are based on a primal-dual interior point algorithm, we prefer solving the dual of the SDP as it results in a smaller internal representation for the SDP solvers ( $\mathbf{Z}$ is treated as a matrix, but $\mathbf{W}$ is parametrized by scalars). We utilize these multipliers to pose the dual of the
SDP relaxation as,

$$
\begin{aligned}
& \max -\sum_{t=1}^{t=T} g_{U}(t)-\sum_{t=1}^{t=T-1} g_{C}(t)+\left(\tau_{i}^{*} b_{i}^{*}-\tau_{i}^{0} b_{i}^{0}\right) \\
& \text { s.t. } i \in \mathcal{N} \text { : } \\
& \left.\begin{array}{l}
h_{W}(t)=-\mathbf{Z}(t), \mathbf{Z}(t) \preceq 0, \\
\bar{\lambda}_{i}^{V}(t), \underline{\lambda}_{i}^{V}(t) \geq 0, \\
i \in \mathcal{N} G
\end{array}\right\} t \in \mathcal{T} \\
& i \in \mathcal{N}^{G} \text { : } \\
& h_{P^{G}}(i, t)=0, h_{Q^{G}}(i, t)=0, \\
& \left.\begin{array}{l}
\bar{\lambda}_{i}^{P}(t), \underline{\lambda}_{i}^{P}(t), \bar{\lambda}_{i}^{Q}(t), \underline{\lambda}_{i}^{Q}(t) \geq 0, \\
\left.\begin{array}{cc}
1 & r_{i}^{1}(t) \\
r_{i}^{1}(t) & r_{i}^{2}(t)
\end{array}\right] \succeq 0,
\end{array}\right\} t \in \mathcal{T} \\
& \bar{\rho}_{i}(t), \underline{\rho}_{i}(t), \bar{\sigma}_{i}(t), \underline{\sigma}_{i}(t) \geq 0, t \in \mathcal{T} /\{T\} \\
& i \in \mathcal{N}^{S} \text { : } \\
& h_{B}(i, 0)=0, \tau_{i}^{0} \text { free, } \tau_{i}^{*} \geq 0, \\
& \left.\begin{array}{l}
h_{B}(i, t)=0, h_{R}(i, t)=0, \\
\bar{\lambda}_{i}^{B}(t), \underline{\lambda}_{i}^{B}(t), \bar{\lambda}_{i}^{R}(t), \underline{\lambda}_{i}^{R}(t) \geq 0 \\
\tau_{i}(t) \text { free, }
\end{array}\right\} t \in \mathcal{T} \\
& (i, j) \in \mathcal{L}: \\
& \left\|r_{i j}^{1}(t), r_{i j}^{2}(t)\right\|_{2} \leq \lambda_{i j}(t), \mu_{i j}(t) \geq 0, \quad t \in \mathcal{T}
\end{aligned}
$$

where $g_{U}(t), g_{C}(t), h_{W}(t), h_{B}(t), h_{R}(t), h_{P^{G}}(i, t), h_{Q^{G}}(i, t)$ are defined in Appendix II.

\section{B. Rank deficiency of SDP relaxations in the single period case}

In [1] the authors presented case studies where the SDP relaxation of the single period OPF problem failed to satisfy the rank condition. These case studies were arrived at by modifying some of the parameters of the standard IEEE test cases (real and reactive power demands, bounds on power generation or line flow limits). The SDP rank condition was also violated non-trivially in some cases, i.e. adding the suggested $10^{-5}$ p.u. perturbation term ([21]) to ensure resistive connectivity of the power network was not sufficient.

We illustrate this with the IEEE 57 bus single period case by considering line flow limits, $S_{i j}^{\max } \forall(i, j) \in \mathcal{L}$, and modifying the demands by a factor $f: f \mathbf{P}^{D}+\mathrm{j} f \mathbf{Q}^{D}$, and varying the resistive perturbation $\delta$ p.u. In the following table, $\left(\lambda_{1}, \lambda_{2}\right)$ denote the largest two eigenvalues of the semidefinite matrix $\mathbf{W}$ and the reported gap is $(U B-L B) / U B * 100$ where $U B$ is the optimal objective value obtained by solving the OPF problem with a local NLP solver and $L B$ is optimal objective value of the SDP relaxation.

\begin{tabular}{|ccccccc|}
\hline$\#$ & $f$ & $S_{i j}^{\max }$ & $\delta$ & $\operatorname{Rank}(\mathbf{W})$ & $\left(\lambda_{1}, \lambda_{2}\right)$ & $g a p$ \\
\hline 1. & 1 & - & 0 & 2 & $(28.76,0.000)$ & $0.000 \%$ \\
2. & 1 & 100 & 0 & 4 & $(28.35,0.009)$ & $0.010 \%$ \\
3. & 1 & 100 & $10^{-4}$ & 4 & $(28.35,0.007)$ & $0.009 \%$ \\
\hline 4. & 1.06 & - & 0 & 2 & $(28.74,0.000)$ & $0.000 \%$ \\
5. & 1.06 & 100 & 0 & 4 & $(28.91,0.049)$ & $3.440 \%$ \\
6. & 1.06 & 100 & $10^{-4}$ & 4 & $(28.91,0.048)$ & $3.447 \%$ \\
\hline
\end{tabular}

TABLE I: Modified test cases 
At the nominal demand ( $f=1)$, the test case satisfied the SDP rank condition. On adding a line flow limit of 100 MVA on all the lines (\# 2), the second largest eigenvalue $\left(\lambda_{2}\right)$ becomes non-negligible and the semidefinite matrix $\mathbf{W}$ violates the rank condition. The constraint on one of the line flow limits is active in this case. Ensuring the network is resistively connected by adding $\delta=10^{-4}$ p.u. perturbation (\# 3) was insufficient to reduce $\lambda_{2}$ to 0 . However, the SDP objective function value was still within $0.01 \%$ of the local solution found using an NLP solver, as indicated by the gap.

When the demands are increased $(f=1.06)$, the SDP rank condition is satisfied in the absence of line flow constraints (\# 4). When a line limit is introduced, $\lambda_{2}$ is not negligible anymore and consequently a gap of $3.44 \%$ results between the NLP local solution and the SDP relaxation (\# 5). The resistive perturbation was not sufficient to reduce $\lambda_{2}$ to 0 in this case as well (\# 6). We refer the interested reader to [23] for an understanding of nonconvexities in the OPF, and [28] for a recent detailed analysis on the same.

\section{GLOBAL OPTIMIZATION BY BRANCH AND BOUND}

The branch and bound method is a general purpose global optimization technique for a wide class of nonconvex problems. It solves the problem $\mathrm{P}$ by constructing a convex relaxation $\mathrm{R}$, that is easy to solve and provides a lower bound $(L)$ on the optimal objective function value (Figure 1a). The upper bound $(U)$ can be arrived at by using local minimization, which also yields a feasible solution. If $U-L$ is sufficiently small, the procedure terminates with the current upper bounding solution. Otherwise, the feasible region is recursively partitioned, and the procedure is repeated (Figure 1b) until the gap $U-L$ is sufficiently small. Nodes are fathomed if the lower bound $L$ is greater than the current best upper bound (Figure 1c). We refer the interested reader to [29] for additional information.

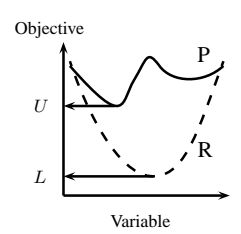

(a) Lower and Upper (b) Bounding

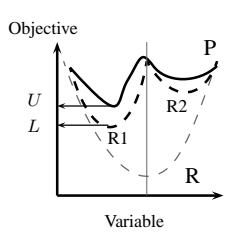

sion

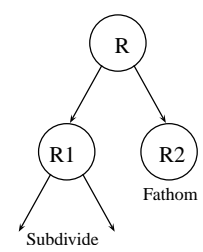

(c) Search tree
Fig. 1: Branch and Bound Schematic

In this work, the upper bounding problem is done through CONOPT [30], a local nonlinear programming (NLP) solver. The lower bounding is done by solving the dual SDP relaxation, implemenented in YALMIP [31]. If an optimality gap exists, the algorithm proceeds by partitioning the feasible region into two sub-regions and repeating the bounding scheme. The partitioning is done as follows. At any node $n$ in the branch and bound tree, define $\forall t \in \mathcal{T}$

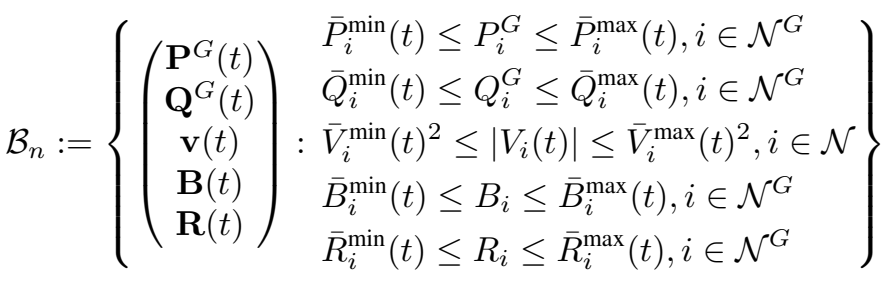

The search space is partitioned by either rectangular bisection on $P_{i}^{G}(t), Q_{i}^{G}(t), B_{i}(t), R_{i}(t)$ or radial bisection on the voltage magnitudes $\left|V_{i}(t)\right|$. The newly generated bounds replace the existing bounds in the upper bounding NLP (2) and lower bounding dual SDP problem (4). The choice of which variable to partition and at which time period is done through a largest violation strategy, i.e. the variable that has the largest difference between the solutions of upper and lower bounding problems is picked to be bisected. For the voltage variables in the lower bounding problem the square root of the diagonal elements of $W(t)$.

We now state the SDP based branch and bound algorithm. ALGORITHM for SDP-BB of MOPF

1. Set BestuB $=$ inf, Best $L B=-i n f$, nodes $=\{0\}$.

2. If (nodes $\neq \emptyset$ and (BestUB - BestLB)/BestUB $>$ tol), choose a live node to explore from nodes. Else return best upper bounding solution as global optimum and STOP.

3. Solve $M O P F(2)$ with bounds $\mathcal{B}_{\text {live }}$

(a) If $M O P F$ is infeasible, fathom live. Go to Step 5.

(b) If $M O P F$ is feasible with objective $z_{\text {live }}^{*}$, update BestUB $=\min \left(\right.$ BestUB, $\left.z_{\text {live }}^{*}\right)$.

4. Solve $S D P(4)$ with bounds $\mathcal{B}_{\text {live }}$.

(a) If $S D P$ is infeasible, fathom live. Go to Step 5.

(b) If $S D P$ is feasible with objective $q_{\text {live }}^{*}$

(i) If $q_{\text {live }}^{*}>$ BestUB or (BestUB $\left.q_{\text {live }}^{*}\right) /$ BestUB $\leq$ tol, fathom live. Go to Step 5.

(ii) Partition $\mathcal{B}_{\text {live }}$ into two regions and update nodes $=\{$ nodes, child1, child2\}. $\mathrm{LB}(\mathrm{child} 1)=\operatorname{LB}(\mathrm{child} 2)=q_{\text {live }}^{*}$. BestLB $=\min (\mathrm{LB}($ nodes $))$. Remove live from nodes.

5. Set BestlB $=\min (\mathrm{LB}($ nodes $))$. Go to Step 2 .

The proof of convergence of this algorithm can be found in [1].

\section{A. Decomposition for large scale MOPF problems}

The efficiency of the above branch and bound algorithm depends strongly on the tightness of the SDP relaxation and also on the computational effort in solving the lower bounding problem. The SDP relaxations of the MOPF tend to be large scale problems and require decomposition methods to address them effectively. We propose a Lagrangian decomposition for the MOPF by dualizing only the time coupled 
constraints (3f), (3g) and (3p) with their dual multipliers,

$$
\begin{aligned}
g^{\mathrm{SDP}}:= & \min \mathrm{L}_{P} \\
\text { s.t. Constraints }(3 \mathrm{~b})-(3 \mathrm{e}),(3 \mathrm{~h})-(3 \mathrm{o}) & \\
& \xi_{\text {in }}(t) \geq 0, \forall t \in \mathcal{T} /\{T\} \\
& \xi_{\text {eq }}(t) \text { free, } \forall t \in \mathcal{T}
\end{aligned}
$$

where $\xi_{\text {in }}(t):=\left(\bar{\rho}_{i}(t), \underline{\rho}_{i}(t), \bar{\sigma}_{i}(t), \underline{\sigma}_{i}(t)\right), \xi_{e q}(t):=\tau_{i}(t)$ and

$$
\begin{aligned}
\mathrm{L}_{P}= & \sum_{t \in \mathcal{T}} \sum_{i \in \mathcal{N}^{S}} \tau_{i}(t)\left(B_{i}(t+1)-B_{i}(t)-\eta R_{i}(t)\right)+ \\
& \sum_{t \in \mathcal{T} /\{T\}} \sum_{i \in \mathcal{N}^{G}}\left[\underline{\rho}_{i}(t)\left(\Delta P_{i}^{\min }-P_{i}^{G}(t+1)+P_{i}^{G}(t)\right)+\right. \\
& \bar{\rho}_{i}(t)\left(P_{i}^{G}(t+1)-P_{i}^{G}(t)-\Delta P_{i}^{\max }\right)+ \\
& \underline{\sigma}_{i}(t)\left(\Delta Q_{i}^{\min }-Q_{i}^{G}(t+1)+Q_{i}^{G}(t)\right)+ \\
& \left.\bar{\sigma}_{i}(t)\left(Q_{i}^{G}(t+1)-Q_{i}^{G}(t)-\Delta Q_{i}^{\max }\right)\right]+\sum_{t \in \mathcal{T}} \sum_{i \in \mathcal{N}^{G}} a_{i}(t)
\end{aligned}
$$

The above objective function (partial Lagrangian) can now be decoupled in time in the space of the primal variables, resulting in $|\mathcal{T}|$ small decoupled SDP's, as opposed to one large SDP. In order to get the best lower bound for MOPF we need to solve,

$$
\max g^{\mathrm{SDP}}\left(\xi_{i n}, \xi_{e q}\right) \text { s.t. } \xi_{i n} \geq 0, \xi_{e q} \text { free }
$$

This is a non-smooth concave maximization problem, which can be solved using a projected subgradient method. At each iteration of the subgradient method $|\mathcal{T}|$ smaller SDP's are solved and the multipliers $\xi_{(\cdot)}$ are updated using a projected subgradient update of the form,

$$
\xi_{e q}^{(k+1)}=\xi_{e q}^{(k)}+\alpha^{(k)} \mathbf{g}_{e q}^{(k)}, \quad \xi_{i n}^{(k+1)}=\left[\xi_{i n}^{(k)}+\alpha^{(k)} \mathbf{g}_{i n}^{(k)}\right]^{+}
$$

There are several ways to choose the stepsize $\alpha^{(k)}$ and search direction $\mathbf{g}^{(k)}$. The method in [32] suggests choosing the step size as $\alpha^{(k)}=\left(q^{(k)}-\hat{q}^{*}\right) /\left\|\mathbf{g}^{(k)}\right\|_{2}^{2}$, where $\hat{q}^{*}$ is an estimate on the best dual value which can be set as $z_{\text {live }}^{*}$, available from Step 3 of the algorithm. The search direction $\mathbf{g}^{(k)}$ is computed through a conic combination of the subgradient and the direction at the previous iteration,

$$
\mathbf{g}^{(k)}=\mathbf{d}^{(k)}+\beta^{(k)} \mathbf{g}^{(k-1)}
$$

with $\beta^{(k)}=\max \left(0,-1.5\left(\mathbf{g}^{(k-1)}\right)^{T} \mathbf{d}^{(k)} /\left\|\mathbf{g}^{(k-1)}\right\|_{2}^{2}\right)$. One element of the subgradient $\mathbf{d}^{(k)}$ is simply the residual of the constraint corresponding to its multiplier in the partial Lagrangian, $\mathbf{d}^{(k)}=\left(\frac{\partial \mathrm{L}_{P}}{\partial \xi_{(\cdot)}}\right)^{(k)}$.

The subgradient method however does not guarantee ascent, and it can take a large number of iterations before the optimal multipliers $\xi_{(\cdot)}$ are found. Hence it is usually terminated after a fixed number of iterations (maxiter). The solution of (6) replaces the solution of (4) in Step 4 of the B \& B algorithm. The performance of the decomposition approach is also reported in the next section.

\section{RESULTS}

We report the results from the branch and bound algorithm applied on the IEEE 57 bus test case with $T=8$ time periods. A time varying demand profile is simulated by scaling the nominal real and reactive power demands on all 57 buses with $f(t)$ as shown in Figure 2, i.e. demands are $f(t)\left(\mathbf{P}^{D}+\mathrm{j} \mathbf{Q}^{D}\right)$ for $t \in \mathcal{T}$. A line limit constraint of

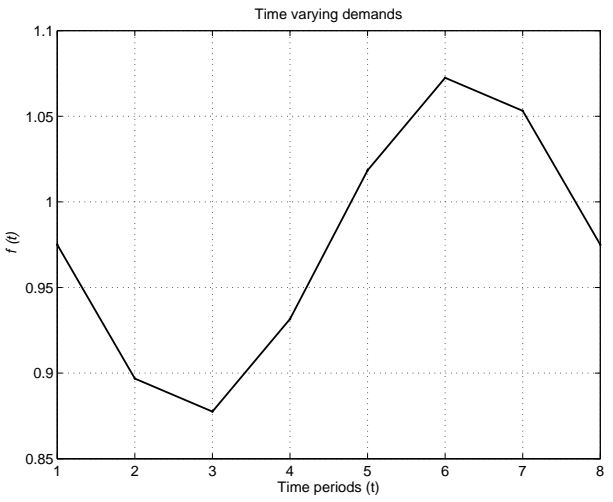

Fig. 2: A sinusoidal time varying demand profile is used as a forecast of the actual demand.

$S_{i j}^{\max }=100$ MVA, $(i, j) \in \mathcal{L}$ is imposed on all the lines. We impose ramp constraints on all the generators of $\Delta P_{i}^{\max }=15$ $\mathrm{MW}, \Delta P_{i}^{\min }=-15 \mathrm{MW}, \Delta Q_{i}^{\max }=15 \mathrm{MVA}$ and $\Delta Q_{i}^{\min }=$ -15 MVA, $\forall i \in \mathcal{N}^{G}$.

We include storage capabilities in buses $\mathcal{N}^{S}=$ $\{5,10,15\}$. The limits on energy storage are set at $B_{i}^{\max }=$ $50 \mathrm{MWh}$ and $B_{i}^{\mathrm{min}}=0 \mathrm{MWh}$. The limits on rate of charging and discharging of the storage system are set at $R_{i}^{\max }=25$ $\mathrm{MW}$ and $R_{i}^{\mathrm{min}}=-25 \mathrm{MW}$, along with a round trip efficiency of $\eta=75 \%$. At initial time $t=1$ the storage is assumed to be fully depleted, i.e. $b_{i}^{0}=B_{i}^{\min }$. The value of the terminal inequality constraint for storage $b_{i}^{*}$, is set to $0.1 B_{i}^{\max }$.

The optimal energy storage policy is shown in Figure 3.

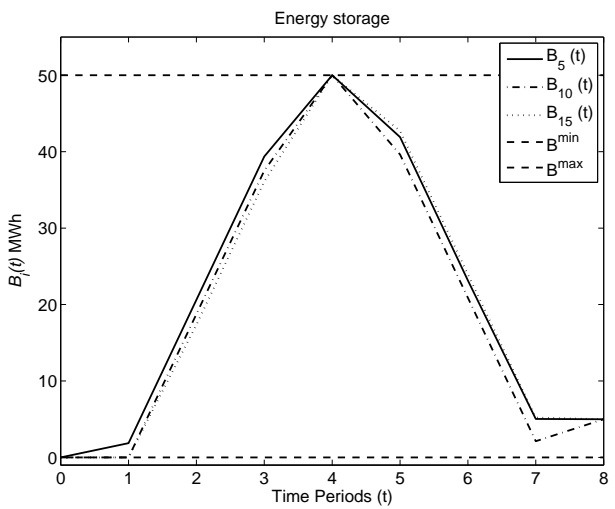

Fig. 3: Optimal energy storage policy $\forall i \in \mathcal{N}^{S}$

During the initial half of the time horizon when the demands are low, energy is generated in excess and stored in the storage systems. The storage systems reach their full capacity at $t=4$. During the latter half of the horizon, the stored energy is used to meet the forecasted higher demands. 
To illustrate the benefits of storage, we compare the above solution with the case without any storage systems $\left(\mathcal{N}^{S}=\right.$ $\{\emptyset\}$ ). For this instance the problem turns out to be infeasible, and in order to obtain a feasible solution the apparent power line limit constraints had to be removed. The globally optimal solution thus computed, violated the line limit of 100 MVA. This demonstrates an additional role that storage systems can play, that of decongesting power lines during peak demands. In the current scenario where transmission capacities are unable to cope up with growth in peak demands, storage can be used to mitigate such congestion related costs as well.

The optimal power generation profiles for generators at buses 1, 3, 6 and 8 are shown in Figure 4 (the other generators operate constantly at their maximum capacity). With
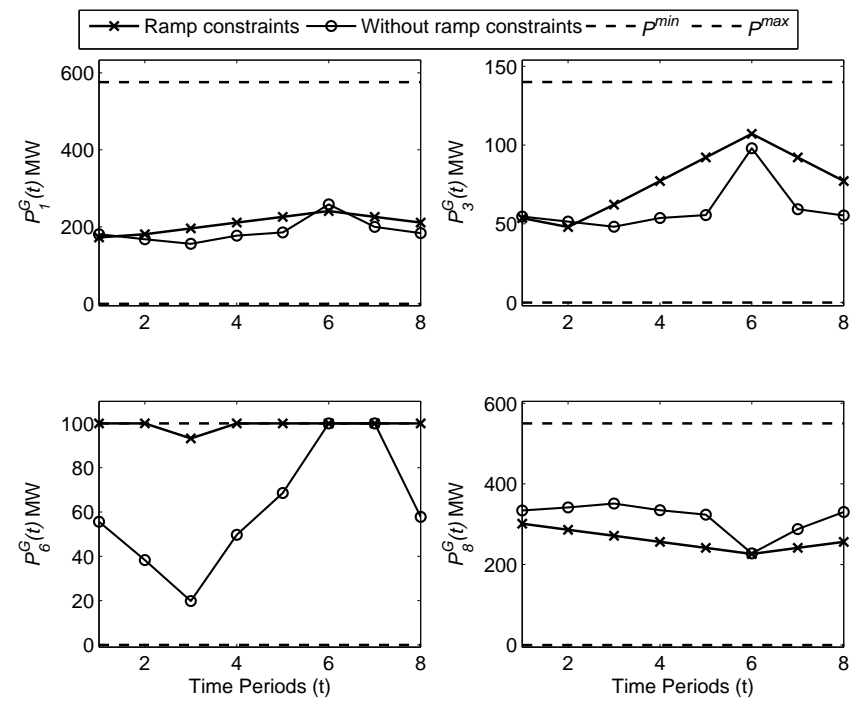

Fig. 4: Real power generation for all $i \in \mathcal{N}^{G}$ both with (cross markers) and without ramp constraints (circle markers)

ramp constraints included (cross markers), the generation profiles are smooth and the energy in the storage systems are gradually built up. Without ramp constraints (circle markers), generators at buses 1, 3 and 6 undergo significant jumps at $t=6$ when the demand is at its highest. A power generation policy with large ramps is not desirable from an operational point of view as it leads to wear and tear and reduction in the lifetime of generators. The ramp constraints thus serve to flatten out power generation profiles in the face of fluctuating demands.

\section{A. Computational experience}

The algorithms were implemented in Matlab 7.10 and executed on a machine with Intel Core i7 $2.8 \mathrm{GHz}$ running under a Windows 7 operating system. All computations were executed on a single processor. For this case study, there is an optimality gap of $2.661 \%$ at the root node. It requires a further 10 nodes in the branch and bound tree to close the optimality gap to within a $1 \%$ tolerance. The following reports the observations when MOPF is solved without any decomposition.
For the MOPF problem even though the SDP rank condition is not always satisfied, the SDP relaxations of the MOPF problem continue to provide strong lower bounds on the globally optimal solution. This is inferred from the low gap at the root node of the B \& B algorithm. However, as the problem size grows, the time taken to solve the SDP relaxations increases significantly. To explore 10 nodes in the branch and bound tree, 283 secs of computational time was required, of which 200 secs (70\%) were spent in solving the SDP's and the $23 \%$ for upper bounding NLP's . When the demand curves were modified slightly, the root node gap was less $(1.6 \%)$ but that case required 31 nodes and a total of 18 minutes to close the gap to $1 \%$ tolerance. Again, close to $75 \%$ of the time was spent in the solution of the SDP's.

It is worthwhile noting that the variable bounds which change from node to node feature only as objective function coefficients (4a) in the dual SDP relaxation. Thus, working with the dual allows us to warm start the SDP's at successive nodes in the B \& B tree using a solution obtained at the parent node (this is a feasible initial point for the child nodes). However, this warm start strategy gave us no additional savings in computational time for this study, as the topic of efficiently warm starting interior point methods is still an open challenge.

\section{B. Performance of the decomposition approach}

When the number of time periods for the above case study was doubled, SeDuMi failed to solve the problem due to memory limitations. In order to test the performance of a Lagrangian dual based decomposition, the method from Section III-A was implemented for the above case study (with 8 time periods). The decomposed subproblems were not solved in parallel. The initial guess for all the multipliers $\xi_{(\cdot)}$ in (6) was chosen as the dual multipliers from the local NLP solver in Step 3 of the algorithm. If (i) the SDP relaxation satisfies the rank condition, and (ii) the local solver finds the global solution at the root node, then such an initialization strategy is guaranteed to close the gap at the root node. In this case study however the root node gap was $3.282 \%$ as the SDP rank condition was violated, and after one hour of computational time, the optimality gap remained the same with the decomposition approach.

The performance of the decomposition strategy is in concordance with the authors' initial observations in [1], that the Lagrangian dual approach is effective in mitigating the computational burden of solving large scale SDP's only for zero duality gap instances of the MOPF. Also, in these instances, taking advantage of parallel computing can provide further speedup. In the non zero gap cases however, it fails to provide strong lower bounds due to the non-smooth nature of the Lagrange dual function and lack of ascent properties in the subgradient algorithm.

\section{CONCLUSion}

In this work, we presented a branch and bound algorithm for global optimization of the multiperiod OPF problem. The time coupling in the MOPF results from storage and ramp 
constraints on generators. An SDP relaxation of the MOPF is used to provide a lower bound for the global optimization algorithm. The solutions indicate that the storage can be effective in offsetting demand variations and also decongesting transmission lines during peak demands. The ramp constraints on the generators serve to flatten out generation profiles. Our algorithm is able to find the globally optimal solution for an 8 time period, 57 bus case with computational times ranging from 5-20 minutes on a standard desktop computer. Most of the computational effort is spent in solving the SDP problems at each node.

\section{A. Future work}

In order to address larger global optimization problems that arise in OPF, it is necessary to seek alternate branch and bound methods that make use of the strength of SDP relaxations, without being affected by their slow solution times. One possible avenue for exploration is the method of [33] to solve the SDP as a semi-infinite LP. At each successive node in the branch and bound tree, semi-definite cuts are generated based on the solution of the LP relaxation along directions that violate the positive semidefiniteness constraint. Another possible avenue is to exploit sparsity in the power networks through matrix completion techniques [34]. It is known that SDP relaxations of nonconvex QCQP's tend to be stronger than SOCP or LP relaxations [35], but very little work has been reported on SDP based branch and bound schemes primarily because the computational burden of solving the SDP relaxations do not make them worthwhile. Also, the lack of efficient warm starting strategies (as opposed to a dual simplex for LP-based branch and bound), make the algorithms scale unfavourably in general. We will investigate alternate solution strategies to mitigate this computational effort.

\section{APPENDIX I}

Following Lavaei and Low [21] we introduce the matrices that are introduced in the definition of the OPF problem below. $\zeta_{i}$ denotes a vector of size $|\mathcal{N}|$ with a 1 at the $i$ th component and zeros elsewhere.

$$
\begin{aligned}
& Y_{\text {bus }, i}:=\zeta_{i} \zeta_{i}^{T} Y_{\text {bus }} \\
& Y_{\text {bus }, i j}:=y_{i j} \zeta_{i} \zeta_{i}^{T}-y_{i j} \zeta_{\zeta} \zeta_{j}^{T} \\
& \left.Y_{i}:=\frac{1}{2}\left[\begin{array}{ll}
\operatorname{Re}\left(Y_{\text {bus }, i}+Y_{\text {bus }, i}^{T}\right) & \operatorname{Im}\left(Y_{\text {bus }, i}^{T}-Y_{\text {bus }, i}\right) \\
\operatorname{Im}\left(Y_{\text {bus }, i}^{T}-Y_{\text {bus }, i}\right) & \operatorname{Re}\left(Y_{\text {bus }, i}+Y_{\text {bus }, i}^{T}\right)
\end{array}\right]\right) \\
& \bar{Y}_{i}:=-\frac{1}{2}\left[\begin{array}{cc}
\operatorname{Im}\left(Y_{\text {bus }, i}+Y_{\text {bus }, i}^{T}\right) & \operatorname{Re}\left(Y_{\text {bus }, i}-Y_{\text {bus }, i}^{T}\right) \\
\operatorname{Re}\left(Y_{\text {bus }, i}-Y_{\text {bus }, i}^{T}\right) & \operatorname{Im}\left(Y_{\text {bus }, i}+Y_{\text {bus }, i}^{T}\right)
\end{array}\right] \\
& Y_{i j}:=\frac{1}{2}\left[\begin{array}{ll}
\operatorname{Re}\left(Y_{\text {bus }, i j}+Y_{\text {bus }, i j}^{T}\right) & \operatorname{Im}\left(Y_{\text {bus }, i j}^{T}-Y_{\text {bus }, i j}\right) \\
\operatorname{Im}\left(Y_{\text {bus }, i j}^{T}-Y_{\text {bus }, i j}\right) & \operatorname{Re}\left(Y_{\text {bus }, i j}+Y_{\text {bus }, i j}^{T}\right)
\end{array}\right] \\
& \bar{Y}_{i j}:=-\frac{1}{2}\left[\begin{array}{cc}
\operatorname{Im}\left(Y_{\text {bus }, i j}+Y_{\text {bus }, i j}\right) & \operatorname{Re}\left(Y_{\text {bus }, i j}-Y_{\text {bus }, i j}^{T}\right) \\
\operatorname{Re}\left(Y_{\text {bus }, i j}-Y_{\text {bus }, i j}^{T}\right) & \operatorname{Im}\left(Y_{\text {bus }, i j}+Y_{\text {bus }, i j}^{T}\right)
\end{array}\right] \\
& M_{i}:=\left[\begin{array}{cc}
\zeta_{i} \zeta_{i}^{T} & 0 \\
0 & \zeta_{i} \zeta_{i}^{T}
\end{array}\right] \\
& M_{i j}:=\left[\begin{array}{cc}
\left(\zeta_{i}-\zeta_{j}\right)\left(\zeta_{i}-\zeta_{j}\right)^{T} & 0 \\
0 & \left(\zeta_{i}-\zeta_{j}\right)\left(\zeta_{i}-\zeta_{j}\right)^{T}
\end{array}\right]
\end{aligned}
$$

\section{APPENDIX II}

The constraints of the SDP dual formulation in (4) are,

$$
\begin{aligned}
& g_{U}(t):=\sum_{(i, j) \in \mathcal{L}}\left[S_{i j}^{\max } \lambda_{i j}(t)+\mu_{i j}(t) L_{i j}^{\max }\right]+\sum_{i \in \mathcal{N}}\left[\alpha_{i}(t) P_{i}^{D}(t)\right. \\
& \left.+\beta_{i}(t) Q_{i}^{D}(t)+\bar{\lambda}_{i}^{V}(t)\left(V_{i}^{\max }\right)^{2}-\underline{\lambda}_{i}^{V}(t)\left(V_{i}^{\min }\right)^{2}\right]+ \\
& \sum_{i \in \mathcal{N}^{G}}\left[\bar{\lambda}_{i}^{P}(t) P_{i}^{\max }-\underline{\lambda}_{i}^{P}(t) P_{i}^{\min }+\bar{\lambda}_{i}^{Q}(t) Q_{i}^{\max }-\underline{\lambda}_{i}^{Q}(t) Q_{i}^{\min }\right]+ \\
& \sum_{i \in \mathcal{N}^{S}}\left[\bar{\lambda}_{i}^{B}(t) B_{i}^{\max }-\underline{\lambda}_{i}^{B}(t) B_{i}^{\min }+\bar{\lambda}_{i}^{R}(t) R_{i}^{\max }-\underline{\lambda}_{i}^{R}(t) R_{i}^{\min }\right]+ \\
& \sum_{i \in \mathcal{N}^{G}}\left[r_{i}^{2}(t)-c_{i 0}\right] \\
& g_{C}(t):=\sum_{i \in \mathcal{N}^{G}}\left[\bar{\rho}_{i}(t) \Delta P_{i}^{\max }-\underline{\rho}_{i}(t) \Delta P_{i}^{\min }+\bar{\sigma}_{i}(t) \Delta Q_{i}^{\max }\right. \\
& \left.-\underline{\sigma}_{i}(t) \Delta Q_{i}^{\min }\right] \\
& h_{W}(t):=\sum_{(i, j) \in \mathcal{L}}\left[r_{i j}^{1}(t) Y_{i j}+r_{i j}^{2}(t) \bar{Y}_{i j}+\mu_{i j}(t) M_{i j}\right]+ \\
& \sum_{i \in \mathcal{N}}\left[-\alpha_{i}(t) Y_{i}-\beta_{i}(t) \bar{Y}_{i}+\bar{\lambda}_{i}^{V}(t) M_{i}-\underline{\lambda}_{i}^{V}(t) M_{i}\right] \\
& h_{P^{G}}(i, t):=\left\{\begin{array}{l}
h_{P G}^{0}(i, t)+\underline{\rho}_{i}(1)-\bar{\rho}_{i}(1), t=1 \\
h_{P^{G}}^{0}(i, t)+\bar{\rho}_{i}(t-1)- \\
\rho_{i}(t-1)-\bar{\rho}_{i}(t)+\rho_{i}(t), t=2 \cdots T-1 \\
\bar{h}_{P^{G}}^{0}(i, t)+\bar{\rho}_{i}(T-1)-\underline{\rho}_{i}(T-1), t=T
\end{array}\right. \\
& h_{P G}^{0}(i, t):=\alpha_{i}(t)+c_{i 1}+2 r_{i}^{1} \sqrt{c_{i 2}}+\bar{\lambda}_{i}^{P}(t)-\underline{\lambda}_{i}^{P}(t) \\
& h_{Q^{G}}(i, t):=\left\{\begin{array}{l}
h_{Q^{G}}^{0}(i, t)+\underline{\sigma}_{i}(1)-\bar{\sigma}_{i}(1), t=1 \\
h_{Q^{G}}^{0}(i, t)+\bar{\sigma}_{i}(t-1)- \\
\underline{\sigma}_{i}(t-1)-\bar{\sigma}_{i}(t)+\underline{\sigma}_{i}(t), t=2 \cdots T-1 \\
h_{Q^{G}}^{0}(i, t)+\bar{\sigma}_{i}(T-1)-\underline{\sigma}_{i}(T-1), t=T
\end{array}\right. \\
& h_{Q^{G}}^{0}(i, t):=\beta_{i}(t)+\bar{\lambda}_{i}^{Q}(t)-\underline{\lambda}_{i}^{Q}(t) \\
& h_{B}(i, t):=\left\{\begin{array}{lr}
\tau_{i}^{0}-\tau_{i}(1), & t=0 \\
\bar{\lambda}_{i}^{B}(t)-\underline{\lambda}_{i}^{B}(t)+\tau_{i}(t)-\tau_{i}^{*}, & t=T \\
\bar{\lambda}_{i}^{B}(t)-\underline{\lambda}_{i}^{B}(t)+\tau_{i}(t)-\tau_{i}(t+1), & \text { else }
\end{array}\right. \\
& h_{R}(i, t):=\bar{\lambda}_{i}^{R}(t)-\underline{\lambda}_{i}^{R}(t)-\alpha_{i}(t)-\eta \tau_{i}(t)
\end{aligned}
$$

\section{REFERENCES}

[1] A. Gopalakrishnan, A. U. Raghunathan, D. Nikovski, and L. T. Biegler, "Global Optimization of Optimal Power Flow Using a Branch \& Bound Algorithm," in Allerton Conference on Communication, Control and Computing, 2012, p. accepted.

[2] T. Ackerman, Wind Power in Power Systems. Wiley, 2005.

[3] J. Carrasco, L. Franquelo, J. Bialasiewicz, E. Galván, R. P. Guisado, M. M. Prats, J. León, and N. Moreno-Alfonso, "Power-electronic systems for the grid integration of renewable energy sources: A survey," IEEE Trans. Industrial Electronics, vol. 53, pp. 1002-1016, 2006.

[4] V. Budhraja, F. Mobasheri, M. Cheng, J. Dyer, E. Castano, S. Hess, and J. Eto, "California's electricity generation and transmission interconnection needs under alternative scenarios," California Energy Commission, Tech. Rep., 2004.

[5] J. P. Barton and D. G. Infield, "Energy storage and its use with intermittent renewable energy," IEEE Trans. Energy Conversion, vol. 19, pp. 441-448, 2004.

[6] S. M. Schoenung, J. M. Eyer, J. J. Iannucci, and S. A. Horgan, "Energy storage for a competitive power market," Annual Review of Energy and the Environment, vol. 21, pp. 347-370, 1996. 
[7] R. Schainker, "Executive overview: Energy storage options for a sustainable energy future," in IEEE Power Eng. Soc. General Meeting, 2004, pp. 2309-2314.

[8] J. W. Tester, E. M. Drake, M. J. Driscoll, M. W. Golay, and W. A Peters, Sustainable Energy: Choosing Among Options. MIT Press, 2005.

[9] J. Carpentier, "Contribution to the economic dispatch problem," Bulletin de la Societe Francoise des Electriciens, vol. 3, no. 8, pp. 431447, 1962, in French.

[10] J. Momoh, M. El-Hawary, and R. Adapa, "A review of selected optimal power flow literature to 1993. Part I: Nonlinear and quadratic programming approaches," IEEE Transactions on Power Systems, vol. 14 , no. 1, pp. 96-104, 1999.

[11] _ _ "A review of selected optimal power flow literature to 1993. Part II: Newton, linear programming and interior point methods," IEEE Transactions on Power Systems, vol. 14, no. 1, pp. 105-111, 1999.

[12] K. S. Pandya and S. K. Joshi, "A survey of optimal power flow methods," J. of Theoretical and Applied Information Technology, vol. 4, no. 5, pp. 450-458, 2008.

[13] R. Jabr, "Radial distribution load flow using conic programming," IEEE Trans. Power Systems, vol. 21, no. 3, pp. 1458-1459, 2008.

[14] _ "A conic quadratic format for the load flow equations of meshed networks," IEEE Trans. Power Systems, vol. 22, no. 4, pp. 2285-2286, 2007.

[15] — "Optimal power flow using an extended conic quadratic formulation," IEEE Trans. Power Systems, vol. 23, no. 3, pp. 1000-1008, 2008.

[16] S. Sojoudi and J. Lavaei, "Network topologies guaranteeing zero duality gap for optimal power flow problem," submitted for publication, 2011

[17] M. Farivar and S. H. Low, "Branch Flow Model: Relaxations and Convexification," http://arxiv.org/abs/1204.4865, 2012.

[18] J. Lavaei, D. Tse, and B. Zhang, "Geometry of Power Flows in Tree Networks," in IEEE PES General Meeting, 2012.

[19] X. Bai, H. Wei, K. Fujisawa, and Y. Wang, "Semidefinite programming for optimal power flow problems," Intl J. of Electrical Power \& Energy Systems, 2008.

[20] X. Bai and H. Wei, "Semidefinite programming-based method for security-constrained unit commitment with operational and optimal power flow constraints," Generation, Transmission \& Distribution, 2009.

[21] J. Lavaei and S. H. Low, "Zero Duality Gap in Optimal Power Flow," IEEE Transactions on Power Systems, in press.
[22] S. Bose, D. Gayme, S. Low, and K. M. Chandy, "Quadratically constrained quadratic programs on acyclic graphs with application to power flow," http://arxiv.org/pdf/1203.5599.pdf, 2012.

[23] B. Zhang and D. Tse, "Geometry of feasible injection region of power networks," http://arxiv.org/pdf/1107.1467.pdf, 2011.

[24] B. Lesieutre, D. Molzahn, A. Borden, and C. L. DeMarco, "Examining the limits of the application of semidefinite programming to power flow problems," in $49^{\text {th }}$ Annual Allerton Conference on Communications, Control and Computing, 2011, pp. 1492-1499.

[25] D. T. Phan, "Lagrangian Duality and Branch-and-Bound Algorithms for Optimal Power Flow," Operations Research, accepted.

[26] D. Gayme and U. Topcu, "Optimal power flow with distributed energy storage dynamics," in American Control Conference, 2011.

[27] J. Warrington, P. Goulart, S. Mariéthoz, and M. Morari, "A market mechanism for solving multi-period optimal power flow exactly on AC networks with mixed participants," in 2012 American Control Conference, 2012, pp. 3101-3107.

[28] W. A. Bukhsh, A. Grothey, K. I. M. McKinnon, and P. A. Trodden, "Local Solutions of Optimal Power Flow," School of Mathematics, University of Edinburgh, Tech. Rep. ERGO-11-017, 2011.

[29] M. Tawarmalani and N. Sahinidis, Convexification and Global Optimization in Continuous and Mixed-Integer Nonlinear Programming. Theory, Algorithms, Software, and Applications, ser. Nonconvex Optimization and Its Applications. Kluwer Academic Publishers, 2002.

[30] A. Drud, "CONOPT - A large scale GRG code," ORSA J. Computing, vol. 6, pp. 207-218, 1994.

[31] J. Löfberg, "Yalmip : A toolbox for modeling and optimization in MATLAB," in Proceedings of the CACSD Conference, Taipei, Taiwan, 2004. [Online]. Available: http://control.ee.ethz.ch/ $\sim$ joloef/ yalmip.php

[32] P. Camerini, L. Fratta, and F. Maffioli, "On improving relaxation methods by modifying gradient techniques." Math. Programming Study, vol. 3, pp. 26-34, 1975

[33] H. D. Sherali and B. M. P. Fraticelli, "Enhancing RLT relaxations via a new class of semidefinite cuts," Journal of Global Optimization, vol. 22, pp. 233-261, 2002.

[34] M. Fukuda, M. Kojima, K. Murota, and K. Nakata, "Exploiting sparsity in semidefinite programming via matrix completion I: General framework." SIAM Journal of Optimization, vol. 11, pp. 647-674, 2000.

[35] P. Kim and M. Kojima, "Second order cone programming relaxation of nonconvex quadratic optimization problems," Optimization Methods and Software, vol. 15, pp. 201-224, 2001 